\title{
Monte Carlo simulation of thermal fluctuations below the onset of Rayleigh-Bénard convection
}

\author{
Jun Zhang and Jing Fan* \\ Laboratory of High Temperature Gas Dynamics, Institute of Mechanics, Chinese Academy of Sciences, Beijing 100190, China
}

(Received 11 January 2009; published 4 May 2009)

\begin{abstract}
The density fluctuations below the onset of convection in the Rayleigh-Bénard problem are studied with the direct simulation Monte Carlo method. The particle simulation results clearly show the connection between the static correlation functions of fluctuations below the critical Rayleigh number and the flow patterns above the onset of convection for small Knudsen number flows $(\mathrm{Kn}=0.01$ and $\mathrm{Kn}=0.005)$. Furthermore, the physical nature for no convection in the Rayleigh-Bénard problem under large Knudsen number conditions (Kn $>0.028)$ is explained based on the dynamics of fluctuations.
\end{abstract}

DOI: 10.1103/PhysRevE.79.056302

PACS number(s): 47.20.Bp, 05.40.-a, 05.70.Ln, 47.45.-n

\section{INTRODUCTION}

Spontaneous small fluctuations always occur in a system at a finite temperature due to molecular thermal motions. Their studies are usually based on the fluctuating hydrodynamics, first proposed in 1957 by Landau and Lifshitz [1]. It is assumed that the fluctuations can be described by the hydrodynamic equations supplemented with random noise terms, which are determined by the fluctuation-dissipation theorem. The theory of fluctuations for fluids in thermodynamic equilibrium is well established [2,3]. For instance, the fluctuation correlations in thermodynamic equilibrium are spatially short ranged, except for states near a critical point.

On the other hand, nonequilibrium fluids, associated with long-range fluctuation correlations, can exhibit a large variety of complex fluctuation phenomena such as thermal convection or turbulence. Thus a study on fluctuations may be the first step in understanding the microscopic mechanisms of macroscopic behavior of fluids. During the past three decades, the fluctuating hydrodynamics has been extended and applied to nonequilibrium fluids based on an assumption that the noise correlations satisfy local thermal equilibrium [4]. As an example, one may consider a fluid confined between two horizontal parallel plates maintained at two different temperatures, which is the well-known Rayleigh-Bénard (R-B) problem. Many studies [4-7] focused on the stationary nonequilibrium states before the convective instability happened, and the coupling between hydrodynamic modes through the nonequilibrium fluxes or gradients was shown to be responsible for the long-range nature of the fluctuations.

Among experimental studies, Sengers and co-workers [8] verified the theoretical analysis of the nonequilibrium contribution to the dynamic structure factor of a liquid. The research group of Ahlers [9] determined the intensity of thermal fluctuations below the onset of R-B convection by shadowgraph technique, and their results were in agreement with the predictions based on fluctuating hydrodynamics.

Since the spontaneous fluctuations are caused by the thermal motions of molecules, a more direct and physically reasonable approach is the particle simulation, such as the direct simulation Monte Carlo (DSMC) method [10,11]. The strat-

\footnotetext{
*Corresponding author; jfan@imech.ac.cn
}

egy of DSMC is to track a large number of representative molecules that move within the computational domain, interact with boundaries, and collide with each other on a probabilistic basis. The DSMC method was first successfully applied to many high-speed problems in context of aerospace engineering, and was then extended to investigate the mechanism of various fundamental flow phenomena at the molecular level, including the R-B transition from thermal conduction to convection [12-14]. Furthermore, it has been applied to study long-range fluctuation correlations in nonequilibrium gases by Mansour et al. [15] and the dynamics of the fluctuations in thermodynamic equilibrium from continuum to collisionless regimes by Bruno et al. [16]. Consequently, the DSMC method is a promising tool to investigate the characteristics of thermal fluctuations, especially, the longrange fluctuations responsible for the self-organization of the complex flows.

In this paper we will employ the DSMC method to study the fluctuations in the R-B problem. As is known, it is the first time that the particle simulation method is used to verify the connection between the fluctuation correlations below the onset of the thermal instability and the flow patterns above the onset of convection. Meanwhile, we want to find out the physical nature why convection cannot happen in the R-B problem with $\mathrm{Kn}>0.028$.

\section{SIMULATION CONDITIONS AND ANALYSIS METHOD}

The relevant nondimensional parameter in the R-B problem is the Rayleigh number [17],

$$
\mathrm{Ra}=\frac{\alpha g \Delta T d^{3}}{\nu \kappa}
$$

where $\alpha, \nu$, and $\kappa$ are the coefficients of volume expansion, kinetic viscosity, and thermal diffusivity, respectively, $g$ is the acceleration of gravity, $\Delta T=T_{l}-T_{u}$ is the temperature difference between the lower and upper plates, and $d$ is the height of the fluid layer. For gas flows discussed here, a substitution of the kinetic transport coefficients for a hardsphere gas into Eq. (1) yields $[13,14]$ 

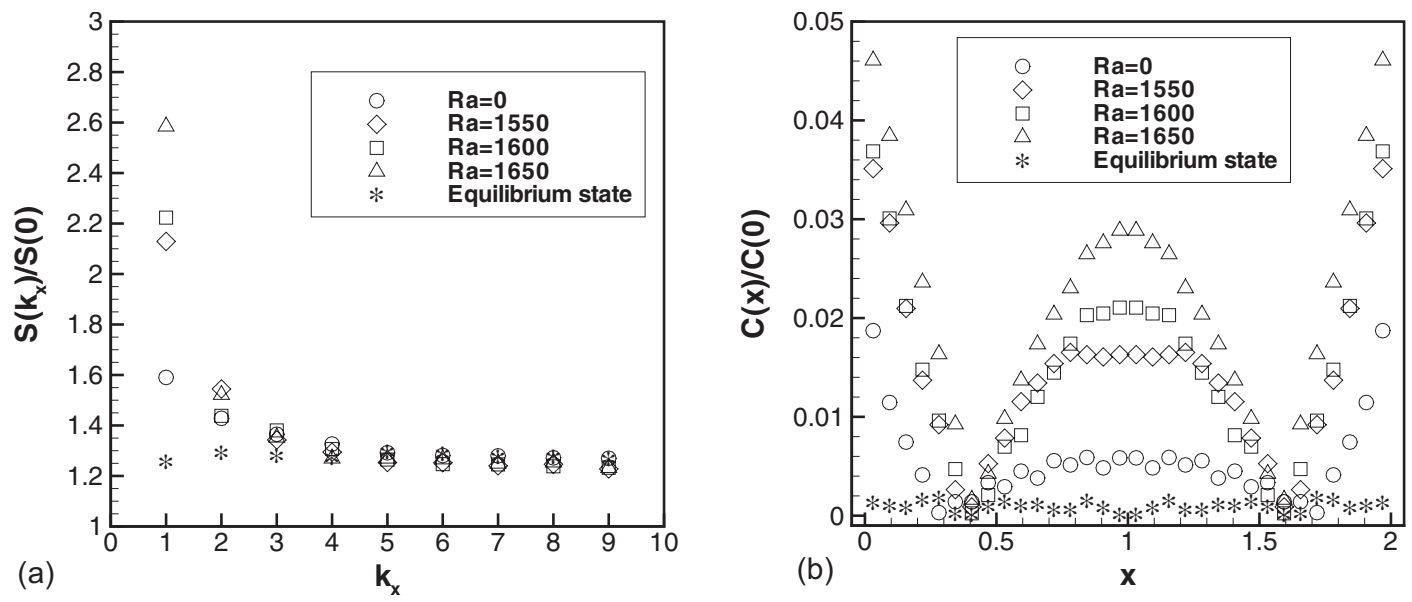

FIG. 1. (a) The static power spectrum and (b) the correlation function of density fluctuations in the horizontal direction for Kn=0.01.

$$
\mathrm{Ra}=\frac{2048}{75 \pi} \times \frac{1-r}{(1+r)^{2} \mathrm{Kn}^{2} \mathrm{Fr}}
$$

It is obvious from Eq. (2) that Ra depends on the temperature ratio $r=T_{u} / T_{l}$, the Knudsen number $\mathrm{Kn}=\lambda / d$, and the Froude number $\mathrm{Fr}=c_{m}^{2} / g d$, where $\lambda$ is the molecular mean-free path and $c_{m}=\sqrt{2 k T_{l} / m}$ is the most probable thermal speed. In the hydrodynamic limit $(\mathrm{Kn} \rightarrow 0)$, thermal convection sets in when $\mathrm{Ra}$ exceeds the critical value at $\mathrm{Ra}_{\mathrm{c}} \cong 1708$ [17]. For finite Knudsen number gas flows, the critical Rayleigh numbers are somewhat different because of "no-Boussinesq" effects $[13,14]$.

In our study we focus on the two-dimensional R-B problem below the onset of thermal convection for a fixed temperature ratio $r=0.1$. The required Rayleigh number is determined by adjusting the Froude number Fr, more concretely, by changing the strength of gravity $[13,14]$. As a usual practice in the DSMC method, the computational domain is divided into sampling cells within which the macroscopic quantities are obtained by sampling the molecular information, and each sampling cell contains some subcells within which collision pairs are selected [10]. The hard-sphere (HS) model is used to describe interactions between gaseous molecules. The calculating time step is smaller than the mean collision time of molecules. Diffusive reflections are assumed at the upper and lower walls, that is, molecules colliding with these walls rebound with an equilibrium Maxwellian distribution at the temperature of the corresponding wall, while periodic boundary conditions are assumed in the horizontal direction. The sampling is taken at the final steady state after an initial transient period. The steady or mean values are obtained by time average in a long enough time duration in sampling cells, while the unsteady or instantaneous values are the average in one calculating time step in sampling cells, then the fluctuations are defined as the difference between the instantaneous and the average values.

In particular, one is usually interested in the power spectrum of density fluctuations of the wave vector $\mathbf{q}$, i.e., the dynamic structure factor $[2,3]$ defined as

$$
S(\mathbf{q}, \omega)=\frac{1}{2 \pi} \int_{-\infty}^{\infty} d t \exp (-i \omega t)\left\langle\delta \rho^{*}(\mathbf{q}, 0) \delta \rho(\mathbf{q}, t)\right\rangle,
$$

where $\delta \rho(\mathbf{q}, t)$ is the space Fourier transform of the density fluctuations and the brackets \langle\rangle denote an ensemble average. Correspondingly, the static characteristics of fluctuation correlations can be described by using the equal-time correlation function, i.e., $C(r)=\langle\delta \rho(0) \delta \rho(r)\rangle$, which is the inverse Fourier transform for the static power spectrum $S(\mathbf{q})$.

In our simulation the density fluctuations are sampled at discrete space-time points. With the boundary conditions being assumed periodic in the horizontal direction, it is easy to determine the static power spectrum of the density fluctuations along the horizontal direction by the Fourier transform on discrete space points at equal time steps, and then the static correlation function can be obtained by the inverse Fourier transform for the static power spectrum. If a double Fourier transform is applied to the density fluctuations on different space and time points, we can obtain the dynamic structure factor.

Since in the DSMC method, each simulated molecule represents a large number of real molecules and the density fluctuations are proportional to the gas density, the intensity of the simulated fluctuations is proportional to the intensity of the real fluctuations. In order to reduce the statistical scatter inherent in a particle simulation method to an acceptable level, long time averaging is performed in each single simulation run.

\section{RESULTS AND DISCUSSION}

\section{A. $K n=0.01$}

The simulation results obtained by the DSMC method show that the critical Rayleigh number $\mathrm{Ra}_{\mathrm{c}}$ based on Eq. (2) for $r=0.1$ is about $1773[13,14]$. First we consider the fluctuations below $\mathrm{Ra}_{\mathrm{c}}$ with $\mathrm{Kn}=0.01$, and the aspect ratio $\Gamma$ $=L / d=2$. The computational domain is divided into 64 $\times 32$ sampling cells, with each containing $4 \times 4$ subcells. We focus our attention on the characteristic of the density fluctuations at the 64 spatial points located at midheight of the 

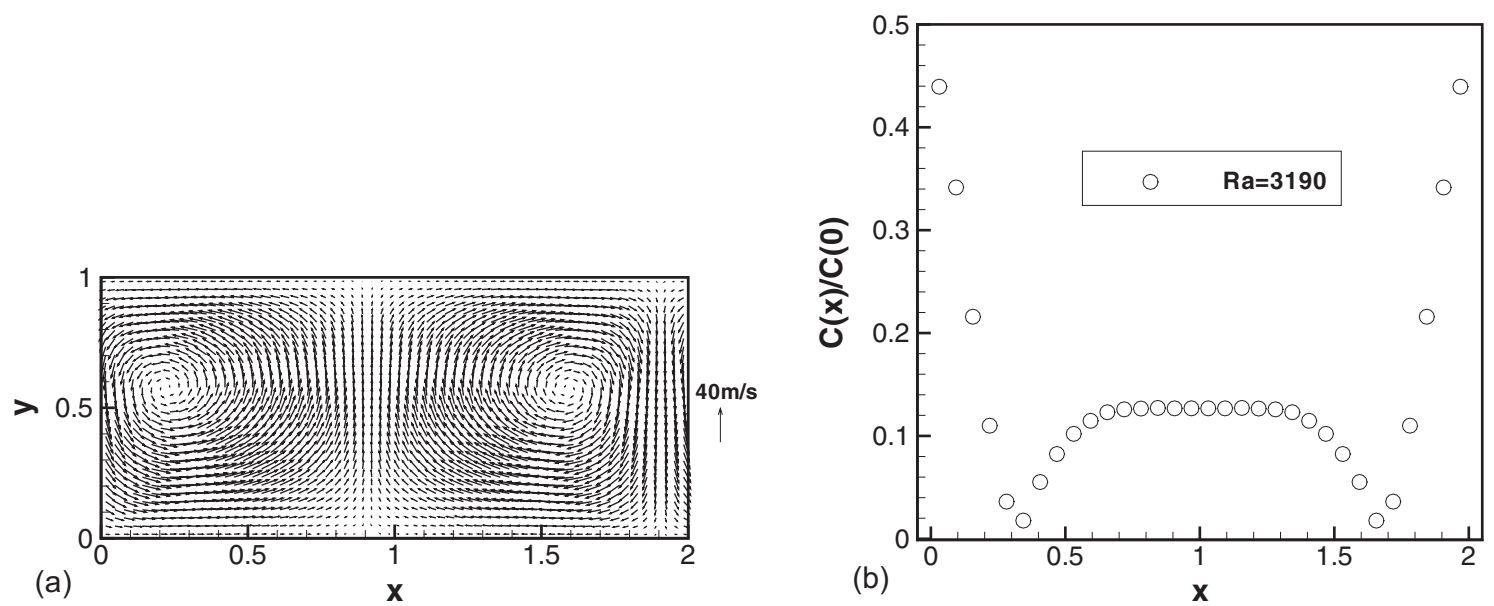

FIG. 2. (a) The velocity vector distributions and (b) the correlation function of density fluctuations in the horizontal direction for Ra $=3190$ and $\mathrm{Kn}=0.01$.

simulation domain. In order to reduce the statistical scatter, we use 10000 samples in long enough time duration to obtain the average. Figure 1 shows the normalized static power spectrum $S\left(k_{x}\right) / S(0)$ and the real-space correlation function $C(x) / C(0)$ for various Rayleigh numbers, where $k_{x}=\frac{q_{x} L}{2 \pi}$ is the discrete wave number in the horizontal direction and $x$ represents the distance between the two space points along the horizontal direction. It is shown that the static power spectrum decreases monotonically with the wave number $k_{x}$ for each Rayleigh number. The corresponding correlation function first decreases with the distance $x$ and then increases until half of the length in the horizontal direction is reached, and it is symmetric about $x=1$. The fluctuation correlation is no longer short ranged like that in thermal equilibrium state, but similar to that of a pair of counter-rotating vortices in a thermal convection (Fig. 2). The result is in agreement with Wu and Alher's experiment [9], which shows that the intensity of fluctuations below the onset of the convection is the maximum at the critical wave number. Figure 1 also shows that the intensity of fluctuation correlations increases as $\mathrm{Ra}$ approaches to $\mathrm{Ra}_{\mathrm{c}}$, as is consistent with the dissipation structure theory founded by Prigogine [18], which indicates that the fluctuation is a trigger mechanism for the phase transition. When the Rayleigh number approaches to its critical value, the random small fluctuations will be strengthened to generate "giant fluctuations" due to coherence effects, then the system changes from an unstable state to a stable and orderly state.

\section{B. $\mathrm{Kn}=0.005$}

If the aspect ratio is fixed as 2.0 and the boundary conditions in the horizontal direction are periodic, there will be only one pair of counter-rotating vortices above the onset of Rayleigh-Bénard convection for $\mathrm{Kn}=0.01$. When $\mathrm{Kn}$ gets smaller, the flow patterns with more vortices may appear [13]. One may ask whether the fluctuations below the onset of convection share all of the physical features of the deterministic patterns that would appear above the threshold. Here we consider the $\mathrm{R}-\mathrm{B}$ problem below $\mathrm{Ra}_{\mathrm{c}}$ for $\mathrm{Kn}=0.005$, and the computational domain is divided into $128 \times 64$ sampling cells, with each sampling cell also containing $4 \times 4$ subcells. It is known that in DSMC method, the molecular movements and intermolecular collisions are uncoupled over short time intervals. Molecular motions are modeled deterministically, whereas intermolecular collisions are handled on a probabilistic basis $[10,11]$. If the random numbers are different, the collision pairs are selected and the postcollision velocities will be different. Therefore, by selecting different random number sequences in different simulation runs, diverse features induced by molecular random thermal motions may be emerged. Figure 3 shows the static power spectrum and the correlation function for $\mathrm{Ra}=1600$. Two cases are considered in almost the same condition except for the random number sequences in the simulation, with the power of $k_{x}=1$ being the maximum in the first case, where the same correlation as of a pair of vortices is obtained, and with the power of $k_{x}$ $=2$ being larger than that of $k_{x}=1$ in the second case, where the correlation as of more vortices is obtained. It is obvious that fluctuations away from the onset of convection foreshadow to some extent all of the possible convective appearance. The earlier theoretical studies based on fluctuating hydrodynamics [19] also show that the nonequilibrium fluctuations close to the R-B instability have an important influence on the pattern-selection process when convection appears, and different characteristics of fluctuations would lead to different convective patterns.

\section{C. $\mathrm{Kn}=\mathbf{0 . 0 5}$}

By using both DSMC and a finite-difference method based on the continuum equations of compressible viscous heat conducting gas with state-dependent transport coefficients, Stefanov et al. [13] obtained the R-B instability zone with respect to two independent parameters $\mathrm{Kn}$ and Fr. The zone of the convection regime was verified by a linear stability analysis of the corresponding continuum equations by Manela and Frankel [20]. Their results indicate the existence of a critical Knudsen number of about 0.028, that is, the thermal convection will not happen when $\mathrm{Kn}>0.028$ even with high Ra numbers. The physical nature can be explained 

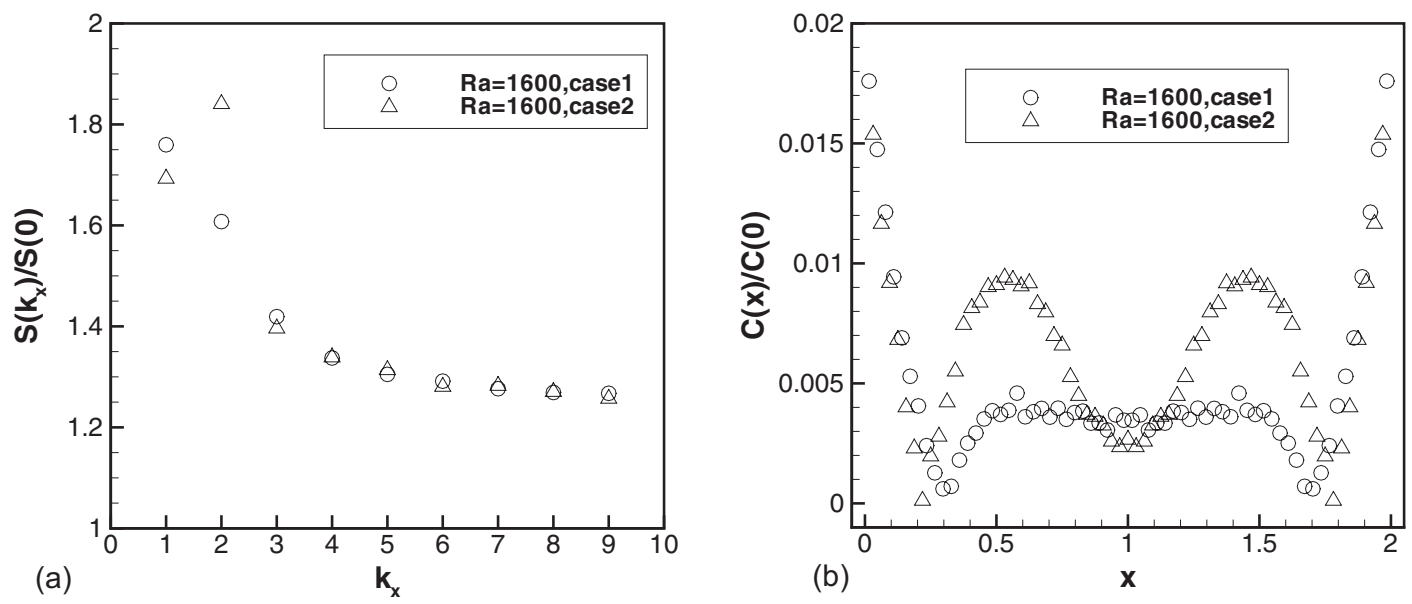

FIG. 3. (a) The static power spectrum and (b) the correlation function of density fluctuations in the horizontal direction for Kn=0.005.

by the characteristics of fluctuations. Let us consider the R-B problem with $\mathrm{Kn}=0.05$ and the aspect ratio $\Gamma=2$. The computational domain is divided into $64 \times 32$ sampling cells, with each sampling cell containing $2 \times 2$ subcells. Figure 4 gives the correlation function of density fluctuations in the horizontal direction for $\mathrm{Kn}=0.05$. In order to facilitate a comparison, the correlation function for $\mathrm{Ra}=1550, \mathrm{Kn}$ $=0.01$, and equilibrium state is reproduced from Fig. 1(b). It obviously shows that there are no long-range fluctuation correlations in the horizontal direction both with $\mathrm{Ra}=1550$ and $\mathrm{Ra}=2900$ for $\mathrm{Kn}=0.05$, as is similar to an equilibrium state but is different from the case of $\mathrm{Kn}=0.01$. Since the longrange fluctuations are the necessary condition for thermal instability, there will be no convection no matter how large the Rayleigh number is for $\mathrm{Kn}=0.05$.

To gain physical insight into the reason why there are no long-range fluctuations in the horizontal direction for $\mathrm{Kn}$ $=0.05$, one can look at the dynamics of the density fluctuations. For simplicity and facilitating statistical calculations, we let $k_{y}=1$ and $k_{x}=0$, i.e., we study the longest-range density fluctuations in the vertical direction, and with space average over the horizontal direction.

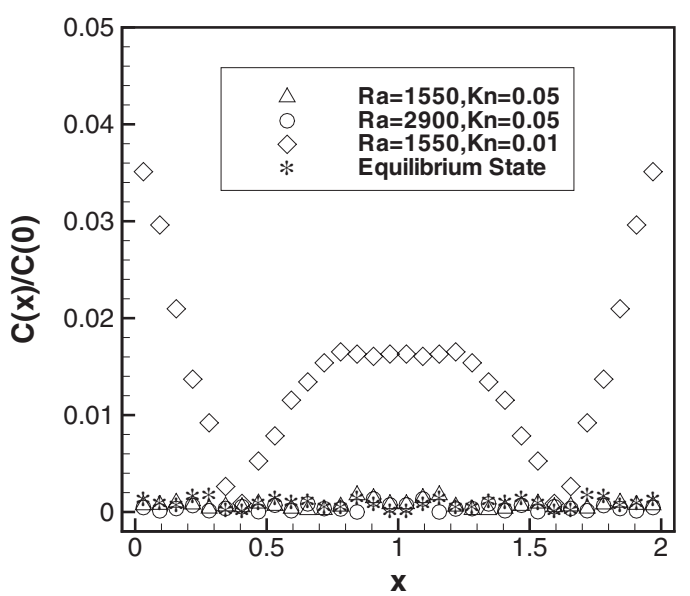

FIG. 4. The correlation function of density fluctuations in the horizontal direction for $\mathrm{Kn}=0.05$. In order to facilitate a comparison, the correlation function for $\mathrm{Ra}=1550, \mathrm{Kn}=0.01$, and equilibrium state is reproduced from Fig. 1(b).
Figure 5 shows the normalized dynamic structure factor $S\left(k_{y}=1, \omega^{*}\right) / S\left(k_{y}=1,0\right)$, where $\omega^{*}=\frac{\omega d}{q_{v} C_{s} N_{t}}, C_{S}$ is the sound speed, and $N_{t}$ is the total time duration. There is a Rayleigh line in the center for $\mathrm{Kn}=0.05$, which corresponds to the thermal diffusivity mode due to temperature fluctuations. Such fluctuations do not propagate and only give rise to pure diffusion. There are Brillouin lines at about $\omega^{*}= \pm 1$, which correspond to the sound modes due to pressure fluctuations coupled with the fluctuating velocity field. The Brillouin doublet is asymmmetrical due to the two associated sound modes propagating through regions of different temperatures. Besides the Rayleigh line and the Brillouin doublet, we can also observe other peaks located at about $\omega^{*}= \pm \frac{1}{2}$. An obvious explanation for these peaks is the existence of standing waves across the system [21]. The density fluctuations may be converted to sound waves, which are reflected by the rigid walls due to the finite size of the system, giving rise to stationary waves. This kind of finite-size effect was also observed in molecular-dynamics computations [22,23]. For $\mathrm{Kn}=0.05$, the intensity of the Brillouin doublet is much smaller than that of the Rayleigh line and the effect of finitesize, meaning that the fluctuations are mainly diffusive and do not propagate. For instance, if there are density or tem-

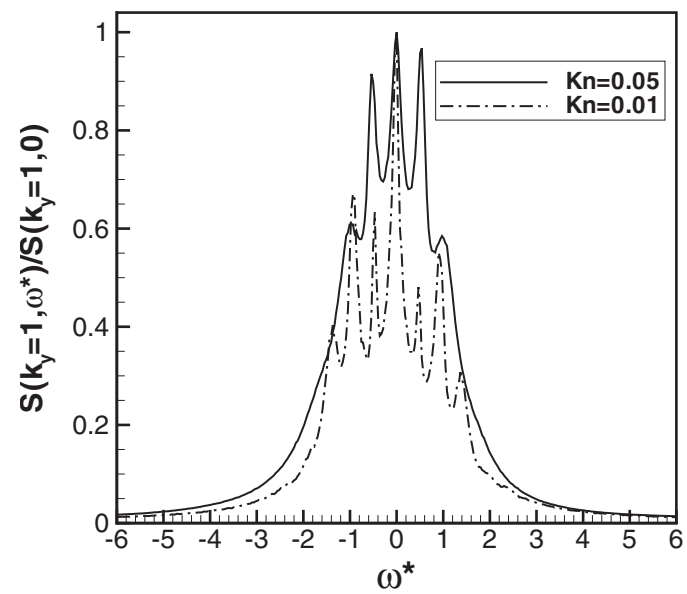

FIG. 5. The dynamic structure factor of the density fluctuations. 
perature fluctuations somewhere in the field, the fluid element will move due to buoyancy and may bring about velocity fluctuations. However, for $\mathrm{Kn}=0.05$ there are only about 20 molecular free paths in the vertical direction, so that particles move almost ballistically between the top and the bottom plates with very little interactions. Therefore, the density or temperature fluctuations in the field are mainly diffusive and hardly result in velocity fluctuations in the vertical direction. This fact reduces the coupling between temperature fluctuations and velocity fluctuations parallel to the temperature gradient, which is responsible for the long-range nature of the fluctuations in the horizontal direction, as shown by fluctuating hydrodynamics [4-7].

For $\mathrm{Kn}=0.01$, the intensity of the Brillouin doublet, centered around $\omega^{*}= \pm 1$, is also smaller than that of the Rayleigh line, but greater than that of the effect of finite-size located at about $\omega^{*}= \pm \frac{1}{2}, \frac{3}{2}$, which means that the effect of the propagating modes of fluctuations plays an important role. Consequently, the density or temperature fluctuations somewhere in the field will cause the velocity fluctuations in the vertical direction. This coupling between the temperature fluctuations and velocity fluctuations brings about long-range fluctuations in the horizontal direction for $\mathrm{Kn}=0.01$. When $\mathrm{Ra}$ approaches to the critical value, the long-range fluctuations will trigger the thermal instability.

\section{CONCLUSIONS}

In this paper we investigate the fluctuations in the R-B problem below the onset of convection with the DSMC method. For small Knudsen numbers, the connection between the fluctuation correlations below the onset of thermal instability and the convective patterns above the threshold is verified. Because the thermal fluctuations are spontaneous due to molecular thermal motions, we can obtain all possible patterns according to the fluctuation correlations although the appearance of the exact pattern is a random event. In fact we can obtain a desired convective pattern by adding external disturbances to strengthen the desired form of fluctuation correlations and suppress other undesired correlations. For instance, by adding a periodic distribution of temperature on the lower plate we can obtain the desired phase and wavelength of the convective rolls. For a large Knudsen number, the fluctuation correlations in the horizontal direction are negligible, thus, the thermal conductive state is always stable. A qualitative explanation is also given based on the dynamics of fluctuations in this paper. Our present observations suggest that the characteristics of the fluctuations below the onset of thermal instability can be used to decide whether there will be convection and to determine the possible flow patterns when convection appears. The next major step will be the study of the fluctuations in complex flows such as the thermal convection or turbulence, which will help us understand the underlying microscopic mechanisms for behaviors of macroscopic fluids.

\section{ACKNOWLEDGMENTS}

This work was supported by the National Natural Science Foundation of China (Grants No. 90205024, No. 10502051, and No. 10621202).
[1] L. D. Landau and E. M. Lifshitz, Fluid Mechanics (Pergamon Press, Oxford, 1959).

[2] J. P. Boon and S. Yip, Molecular Hydrodynamics (McGrawHill, New York, 1980).

[3] B. J. Berne and R. Pecora, Dynamic Light Scattering (Wiley, New York, 1976).

[4] J. M. Ortiz de Zárate and J. V. Sengers, Hydrodynamic Fluctuations in Fluids and Fluid Mixtures (Elsevier, Amsterdam, 2006).

[5] D. Ronis and I. Procaccia, Phys. Rev. A 26, 1812 (1982).

[6] R. Schmitz and E. G. D. Cohen, J. Stat. Phys. 39, 285 (1985).

[7] B. M. Law and J. V. Sengers, J. Stat. Phys. 57, 531 (1989).

[8] B. M. Law, R. W. Gammon, and J. V. Sengers, Phys. Rev. Lett. 60, 1554 (1988); B. M. Law, P. N. Segrè, R. W. Gammon, and J. V. Sengers, Phys. Rev. A 41, 816 (1990); P. N. Segrè, R. W. Gammon, J. V. Sengers, and B. M. Law, ibid. 45, 714 (1992).

[9] M. Wu, G. Ahlers, and D. S. Cannell, Phys. Rev. Lett. 75, 1743 (1995); J. Oh and G. Ahlers, ibid. 91, 094501 (2003).

[10] G. A. Bird, Molecular Gas Dynamics and Direct Simulation of Gas Flows (Clarendon, Oxford, 1994).

[11] C. Shen, Rarefied Gas Dynamics: Fundamentals, Simulations and Micro Flows (Springer, Berlin, 2005).

[12] A. Garcia, in Microscopic Simulation of Complex Flows, edited by M. Mareschal (Plenum, New York, 1990), p. 177; A.
Garcia and C. Penland, J. Stat. Phys. 64, 1121 (1991).

[13] S. Stefanov, V. Roussinov, and C. Cercignani, Phys. Fluids 14, 2255 (2002); 14, 2270 (2002); 19, 124101 (2007).

[14] J. Zhang and J. Fan, Chin. Sci. Bull. 54, 364 (2009).

[15] M. M. Mansour, A. L. Garcia, G. C. Lie, and E. Clementi, Phys. Rev. Lett. 58, 874 (1987).

[16] D. Bruno, M. Capitelli, S. Longo, and P. Minelli, Chem. Phys. Lett. 422, 571 (2006).

[17] S. Chandrasekhar, Hydrodynamic and Hydromagnetic Stability (Clarendon, Oxford, 1961).

[18] G. Nicolis and I. Prigogine, Self-organization in Nonequilibrium Systems: From Dissipative Structures to Order Through Fluctuations (Wiley, New York, 1977).

[19] V. Zaitsev and M. Shliomis, Sov. Phys. JETP 32, 866 (1971); R. Graham, Phys. Rev. A 10, 1762 (1974); J. B. Swift and P. C. Hohenberg, ibid. 15, 319 (1977).

[20] A. Manela and I. Frankel, Phys. Fluids 17, 036101 (2005).

[21] D. Gutkowicz-Krusin and I. Procaccia, Phys. Rev. Lett. 48, 417 (1982); G. Satten and D. Ronis, Phys. Rev. A 26, 940 (1982).

[22] M. Malek Mansour, A. L. Garcia, J. W. Turner, and M. Mareschal, J. Stat. Phys. 52, 295 (1988).

[23] M. Mareschal, M. M. Mansour, G. Sonnino, and E. Kestemont, Phys. Rev. A 45, 7180 (1992). 